\section{Resolution Optical Tomography for Imaging of Biological Tissues}

Imaging of soft biological tissues using noninvasive techniques is of crucial interest in biomedicine as a satisfactory technique would greatly aid in detecting abnormalities and functions within such tissues. In the December 1, 2004, issue of Optics Letters (p. 2770), S. Sakad囚ic and L.V. Wang (Optical Imaging Laboratory at Texas A\&M University) have reported the realization of high-resolution ultrasound-modulated optical tomography based on optical contrast for imaging soft biological tissues.

Ultrasound-modulated optical tomography is a hybrid technique that combines ultrasonic resolution with optical contrast. Ultrasound generates collective motions of the optical scatterers in a tissue and also leads to periodic changes in the optical index of refraction. This leads to fluctuations in the intensity of the speckles formed by multiple scattered light. Thus, the ultrasound-modulated component of light contains information about the tissue from the region of interaction between the optical and ultrasonic waves. Measurement techniques such as parallel speckle detection combined with ultrasound frequency sweep and computer tomography are commonly used to detect the modulated component of light produced by continuous-wave ultrasound. However, pulsed ultrasound provides direct resolution along the optical axis and is more compatible with conventional ultrasound imaging.

In SakadXic and Wang's study, a longcavity confocal Fabry-Perot interferometer (CFPI) is used to achieve high-resolution ultrasound-modulated optical imaging. A CFPI is advantageous due to its high etendue, which is defined as the geometric capability of an optical system to transmit radiation; more specifically, it is the product of the opening size and solid angle from which the system accepts light. The CFPI is able to detect the propagation of high-frequency ultrasound pulses in real time and tolerate speckle decorrelation. Ultrasound frequency of $15 \mathrm{MHz}$ was used to detect $\sim 100-\mu \mathrm{m}$-sized objects placed $\sim 3 \mathrm{~mm}$ below the surface of chicken breast tissue samples. Two chicken breast tissue samples were investigated, each with a radius of curvature of $\sim 3 \mathrm{~mm}$.

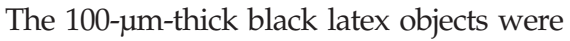
placed in the centers of curvature of the samples, with their wide sides parallel to the ultrasound beam. As shown in Figure 1, estimated axial and lateral resolutions of $70 \mu \mathrm{m}$ and $120 \mu \mathrm{m}$ were measured, respectively. The researchers said this study demonstrates the feasibility of
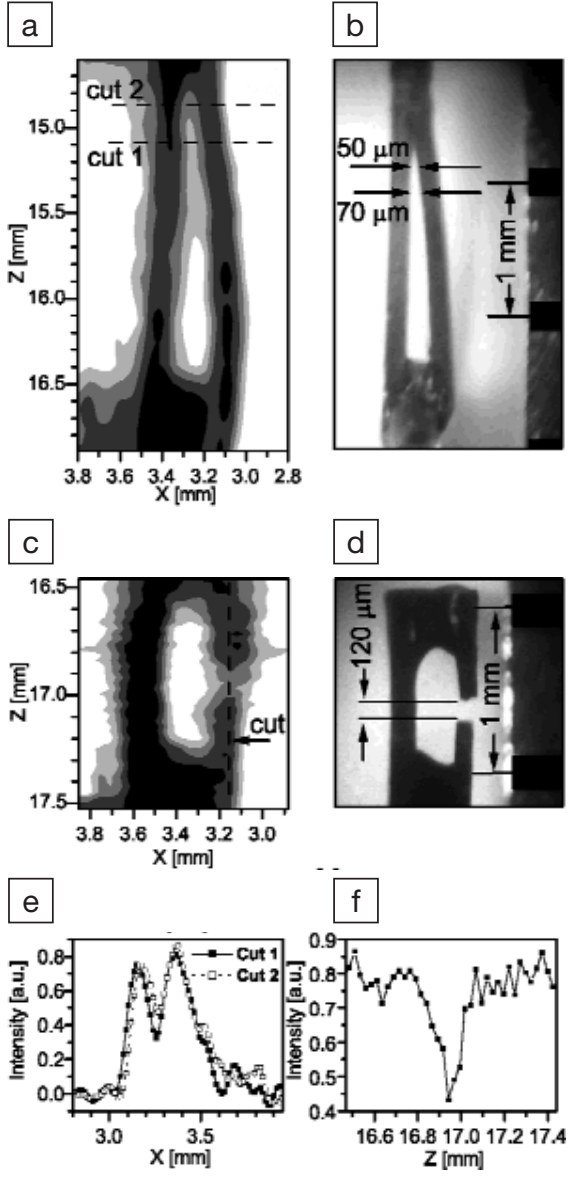

Figure 1. Measurement of the axial and lateral resolutions in breast tissue. (a) Measurement and (b) image of an object, showing the axial resolution. (c) Measurement and (d) image of an object, showing the lateral resolution. (e) Onedimensional (1D) axial profiles of intensity from the data in (a). (f) $1 D$ lateral profile of intensity from the data in (c). Reproduced with permission from Optics Letters 29 (23) (December 1, 2004), p. 2772. (C) 2004 Optical Society of America.

high-resolution ultrasound-modulated optical tomography for imaging biological tissue to a depth of several millimeters. The researchers also said that the resolution can be improved upon by using higher ultrasound frequencies.

ADITI S. RISBUD

\section{Direct Methanol-Air Fuel Cell with Nanoporous Proton-Conducting Membrane Reaches Cell Power of $0.5 \mathrm{~W} / \mathrm{cm}^{2}$}

Fuel cells are attracting considerable attention for possible applications in portable electronics, distributed energy, and transportation. Direct methanol fuel cells composed of nanoporous proton- conducting membranes can be used for these applications because of their lower cost, higher conductivity, higher water permeation under hydraulic pressure, and lower fuel crossover as compared with a commercial counterpart, a Nafion membrane. In the December 2004 issue of Electrochemical and Solid-State Letters (p. A507), E. Peled (Tel-Aviv University), M. Rakhman (Israel Plastics and Rubber Center in Haifa), and their colleagues report on the performance of a nanoporous protonconducting membrane (NP-PCM)-based direct methanol fuel cell (DMFC) operated with triflic acid instead of sulfuric acid. The composition of the NP-PCM was $28 \mathrm{vol} \%$ poly(vinylidene fluoride), $12 \mathrm{vol}^{\%} \mathrm{SiO}_{2}$, and $60 \mathrm{vol} \%$ liquid (an acid-methanol mixture. It was hot-pressed at $100^{\circ} \mathrm{C}$ and $24 \mathrm{~kg} / \mathrm{cm}^{2}$ to a final thickness of 70-100 $\mu \mathrm{m}$. The purpose of the triflic acid, in contrast to sulfuric acid, is that it does not adsorb on the platinum catalyst. In conjunction with a membrane with high water permeability and high conductivity, a fuel cell is produced that can increase power by up to $250 \%$, as compared with sulfuric acidbased systems. In fuel cells in which sulfuric acid is used, a higher overpotential at both electrodes is observed, while switching to triflic acid decreases the overpotential at the anode from $0.47 \mathrm{~V}$ to $0.33 \mathrm{~V}$, and the overpotential at the cathode drops from $0.44 \mathrm{~V}$ to $0.36 \mathrm{~V}$. This decrease in overpotenial leads to a rise in cell voltage, which produces a maximum power density $\left(0.5 \mathrm{~W} / \mathrm{cm}^{2}\right)$ at $130^{\circ} \mathrm{C}$. This particular fuel cell has shown an acceptable operating lifetime of $1000 \mathrm{~h}$ at room temperature and is efficient when compared with reformate-hydrogen fuel cells. The direct methanol fuel cell studied by Peled's group competes favorably with indirect methanol or gasoline fuel cells when size, weight, complexity, and efficiency are considered.

LARKEN E. EULISS

\section{Copper Nanowires Broken into Nanospheres by Rayleigh Instability}

Despite significant progress in understanding materials behavior at the macroscopic level, the behavior of small-scale nanomaterials under changing conditions remains largely unknown. However, this behavior must be addressed before new small-scale materials can be successfully used in technological applications. M.E. Toimil Molares (Gesellschaft für Schwerionenforschung, Darmstadt, Germany), A.G. Balogh (Technische Universität Darmstadt), and their colleagues have reported significant progress in understanding the behavior of small volumes of copper in the shape 